\title{
Signal Game Analysis on the Effectiveness of Coal Mine Safety Supervision Based on the Affective Events Theory
}

\author{
Xue Yang, ${ }^{1}$ Yang Tian, ${ }^{1}$ Kai Feng $\mathbb{D}^{1},{ }^{1}$ Juan Yang, ${ }^{1}$ Shu-hui Zhang, ${ }^{1}$ and Shengnan Wang ${ }^{2}{ }^{2}$ \\ ${ }^{1}$ School of Management and Economics, North China University of Water Resources and Electric Power, \\ Zhengzhou 450046, China \\ ${ }^{2}$ Institute of Psychology and Behavior, Henan University, Kaifeng 475000, China \\ Correspondence should be addressed to Kai Feng; fengkai923@hotmail.com and Shengnan Wang; nicolesnwang@163.com
}

Received 2 January 2020; Accepted 4 June 2020; Published 26 June 2020

Academic Editor: Qingling Wang

Copyright (C) 2020 Xue Yang et al. This is an open access article distributed under the Creative Commons Attribution License, which permits unrestricted use, distribution, and reproduction in any medium, provided the original work is properly cited.

\begin{abstract}
The main cause of coal mine safety accidents is the unsafe behavior of miners who are affected by their emotional state. Therefore, the implementation of effective emotional supervision is important for achieving the sustainable development of coal mining enterprises in China. Assuming rational players, a signaling game between miners (emotion-driven and judgement-driven) and managers is established from the perspective of Affective Events Theory in order to examine the impact of managers' emotions on coal miners' behavior; it analyzes the players' strategy selections as well as the factors influencing the equilibrium states. The results show that the safety risk deposits paid by managers and the costs of emotion-driven miners disguising any negative emotions affect equilibrium. Under the separating equilibrium state, the emotional supervision system faces "the paradox of almost totally safe systems" and will be broken; the emotion-driven miners disguising any negative emotions will be permitted to work in the coal mine, creating a safety risk. Under the pooling equilibrium state, strong economic constraints, such as setting suitable safety risk deposits, may achieve effective emotional supervision of the miners, reducing the safety risk. The results are verified against a case study of the China Pingmei Shenma Group. Therefore, setting a suitable safety risk deposit to improve emotional supervision and creating punitive measures to prevent miners from disguising any negative emotions can reduce the number of coal mine safety accidents in China.
\end{abstract}

\section{Introduction}

Emotions can affect people in many aspects of their lives and define a person's happiness, stress, and longevity $[1,2]$. In high-risk industries, such as coal mining, emotions directly cause unsafe behavior [3] and are, thus, an "invisible killer" that is detrimental to the life safety of employees. Scholars have found that people's emotions are important contributing factors to the social production of safety [4-6] and are caused by various affective events in an employee's work and life [7]. Research in the field of organizational behavior has stated that affective events inevitably occur when employees interact with colleagues and leaders during work [8,9]. Weiss and Cropanzano [10] proposed the Affective Events Theory (AET) to explore the relationships between affective events and reactions and between attitudes and behaviors of organization members. Scholars have since conducted a series of verifications that developed AET $[9,11,12]$. AET provides a natural support for the study on the induction and prevention of emotions that create an unsafe environment from a dynamic perspective but has not garnered enough attention in the field of safety management. Few scholars have tried to analyze the sources of unsafe emotions in the workplace from the perspective of AET [13].

Coal consumption accounts for more than $60 \%$ of total energy consumption in China, reflecting the importance of coal mine safety production in one of the pillar industries that play a leading role in China's economic development. Safety management in China differs significantly from those in developed countries. Developed countries have mature production management systems due to their high levels of economic development. In addition, employees in developed 
countries have a higher average educational level, stronger selfsafety awareness, and better control over their emotions than those in developing countries do. Under these conditions, enterprise safety management is based on the individual psychological motivations of employees and attaches the importance to a human-centered, flexible enterprise safety performance management [14]. While China has experienced rapid economic development during the 30 years of reform after opening up, there still exist gaps in safety management between it and developed countries. In China, while production management and management systems have gradually improved, its employees' educational level and safety awareness remain significantly different from those in developed countries. Additionally, Chinese employees generally express emotions more implicitly than do their counterparts in Western countries [15]. Under these conditions, enterprise safety management is based on the system, culture, and atmosphere and attaches importance to a system-centered, fixed management of individual safety behaviors [16, 17]. Therefore, under a system-centered, fixed management, improving the safety management of Chinese coal mining enterprises is critically dependent upon effectively suppressing the inducing effect of internal factors, such as emotions, on unsafe behaviors and establishing the causes and countermeasures of coal mine safety accidents.

Given the current conditions of coal mine production in China, a "miner-manager" signal game model is constructed from the perspective of AET. By calculating and analyzing the conditions of separating equilibrium and pooling equilibrium, possible suggestions for improving miners' emotional supervision are provided.

\section{Literature Review}

The work conditions and environment in China's high-risk industries are based on the nature of production. In particular, the coal mining industry operates in small spaces underground under the harsh conditions of high temperatures and humidity and is constantly changing because of the mining process. The requirements for safety are also extremely demanding. The government has always focused on the supervision of coal mine safety production. For example, in 2018, General Secretary Xi Jinping set eight important instructions on coal mine safety management [18], three of which addressed coal mine safety production.

Studies have shown that anxiety, anger, and even excessive happiness can lead to the unsafe behavior of employees [19-21] and may lead to safety accidents, but a clear, common definition of such emotions has not been found. Based on the existing research, the emotions that may lead to employees performing unsafe behavior are hereafter defined as "unsafe emotions."

The research that addressed preventing unsafe emotions generally followed the logic of "emotional recognition/ measurement-unsafe behavior prevention" [22-24]. Although reasonable, it is actually the prevention of unsafe behavior, not unsafe emotions, that is lacking. In fact, the research on preventing unsafe behavior is lagging behind that of preventing unsafe emotions. Existing research is based on the assumption that emotional outbursts, or sudden changes in mood, can, to some extent, be identified by instrument or visual observations. However, latent emotions are difficult to observe and monitor. As it has been recorded that, culturally, the emotional expressions of people in China are generally implicit, the abovementioned research limitations are relevant to this study. Thus, it is crucial to design a new theoretical path.

Scholars have studied the influence mechanisms of emotions on individual behavior from different perspectives. These theories can be divided into two categories, depending on how emotions affect behavior. One category is based on emotional contagion, which is the idea that emotions are transmitted from one person to another and that people subconsciously copy other people's facial expressions, sounds, postures, and movements simultaneously. The length of the imitation of these emotional characteristics is very short and almost synchronous $[25,26]$. Such research is represented by the emotional contagion theory (ECT) [27]. The other category is based on individual cognitive appraisal, which believes that the influence of emotion on individual behavior is not through simple emotional contagion but the result of individual processing and judgement of the emotional information from others. In other words, when in the presence of others' emotions, individuals may show different emotional or behavioral characteristics [28-30]. Such a phenomenon is known as Cognitive Appraisal Theory of Emotion (CATE) [31].

However, further studies found that the impact of others' emotions on an individual's behavior may be driven by both emotional contagion and cognitive appraisal [10], which may be integrated. Thus, AET was proposed. Compared to ECT and CATE, AET has advantages in scope and explanatory power. Therefore, this paper conducts research based on AET.

AET is gradually emerging in the field of organizational behavior and naturally provides new methods for solving the abovementioned dilemmas. AET considers the dynamic nature of emotions and believes that the characteristics of the work environment and work events, for example, affect the emotional response of employees and, thus, their behavior (see Figure 1). For example, the simple and tedious nature of work tasks can easily provoke employees' emotional reactions of being bored [32]. Likewise, challenging events can stimulate an employee's attentiveness emotional response, and interrupting events can trigger an indignation emotional reaction [33]. A leader's behavior and leadership style can also result in different emotional reactions from their employees [34]. Thus, in terms of the impact of emotional responses on behavior, AET proposes two types of behaviors, affect-driven and judgement-driven, although the theory applies an effective response rather than an emotional response (emotional responses in actual research are often measured by emotions) [35].

AET is based on the "symmetry assumption" [36]; that is, the leader's negative emotion leads to a negative emotional event. AET is focused on explaining the negative influence of the leader's negative emotion on employee performance and has obvious limitations in explaining the positive effects of leaders' negative emotions [37]. 


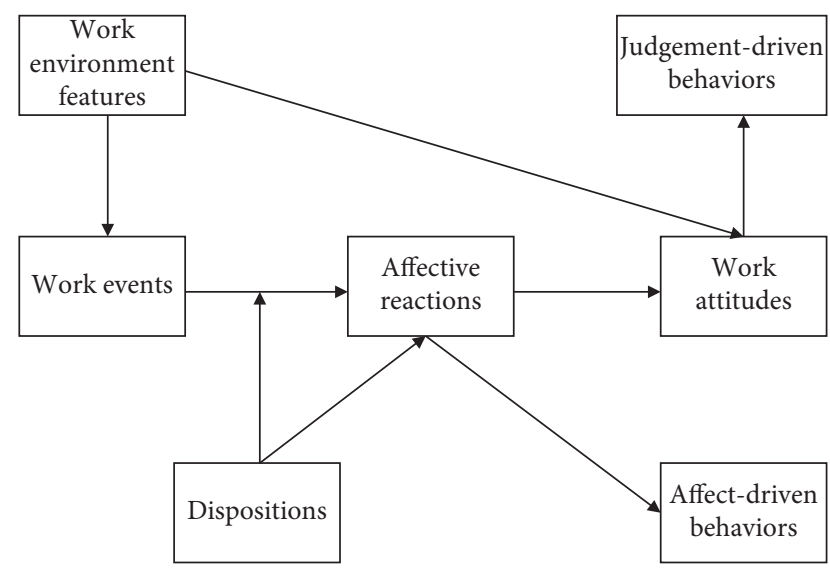

FIgURE 1: Schematic diagram of macroscopic structure of Affective Events Theory.

On the basis of AET, Van Kleef introduced the idea of information processing in the study of leader's emotions and developed the Emotion As Social Information (EASI) model [38], which indicates that observers choose a path, affective reactions, or inferential processes through the judgements they make from emotional expressions. In the context of the coal miners, employees who choose the affective reactions path, or emotional contagion, are infected with the leader's negative emotions, resulting in reduced performance; employees who choose the inferential processes path, or cognitive appraisals, will extract information about the completion of the employee's task from the leader's negative emotions and respond by improving their performance (see Figure 2).

From the above discussion, we can find that although the existing researches have greatly promoted the development of AET by providing a theoretical explanation of the impact of affective events on employee behavior, most of them are based on the perspective of employees. There are few studies on how managers conduct effective emotional supervision for different types of employees. As such, the existing research does not address the following two questions well:

Question 1: with the continuous improvement of hardware conditions in coal mine safety production, such as supporting technology and equipment, the safety status of coal mine production has been significantly improved. However, the safety accidents caused by the unsafe behavior of miners still prevail, and emotion is the direct cause of this unsafe behavior. How can the causes and countermeasures for the repeated prohibitions of coal mine safety accidents be found from the emotional level?

Question 2: does the safety risk mortgage incentive mechanism implemented by coal mines affect the emotional supervision of miners? How can the amount of safety risk deposit be set to achieve effective supervision of miners' emotions?

In order to answer the above two questions, this paper introduces the AET in the field of organizational behavior. It combines the actual reward and punishment system of coal mines to construct the "miner-manager" signal game model from the perspective of affective events and ultimately provides possible advice on the efficiency of miners' emotional supervision.

\section{Model Assumptions and Signal Game Model Construction}

\subsection{Model Assumptions}

(1) Due to economic reasons, the expected return of miners' downhole operations is positive. Only under this assumption do the miners have the motivation to work underground, and the coal mining enterprises operate.

(2) Referring to Hsieh's method, we divide miners into two categories: miners who are easily driven by emotion (emotion-driven miners) and miners who are driven by cognitive appraisal (judgement-driven miners) [39].

(3) If emotion-driven miners are affected by the negative emotions of the leader and, as a result, are prohibited from downhole work, the miners' psychological damage will be denoted as $X$. As they have a negative outlook, they will think that $X$ is bigger than the fixed wage $W: X>W$.

(4) According to the EASI model, miners who choose emotional contagion experience a reduction in safety performance, while miners who choose cognitive appraisal improve safety performance. It is assumed that the miner who chooses the latter improves their safety performance to such an extent that they do not cause a safety accident.

3.2. Game Subject. Before the miners go downhole to work, they have a prework meeting during which tasks are assigned and the managers assess their mental and physical states. The miners who fail this assessment are asked to take steps to improve. However, if the miners continue to not meet the requirements for downhole work, they are prohibited from doing so by the managers. In summary, it is assumed that Player 1 of the game represents the miners who are preparing for downhole work and are divided into emotiondriven miners $k^{E}$ and judgement-driven miners $k^{D}$. The miners' classification into emotion-driven and judgementdriven miners is determined by questionnaires in conjunction with their daily performance. Player 2 represents the managers. Both players behave rationally; they have the ability to make optimal response strategies under given conditions to maximize their own interests.

3.3. Game Signal. Before the miners are lowered into the mine, the managers conduct a safety status judgement on each miner. To be able to work downhole, the miners may disguise their emotional state. Therefore, the emotional state $(m)$ exhibited by the miner is the signal in the signal game model and forms the signal space $M=\left\{m^{N}, m^{P}\right\}\left(m^{N}\right.$ represents a stable emotional state, and $m^{P}$ represents a 


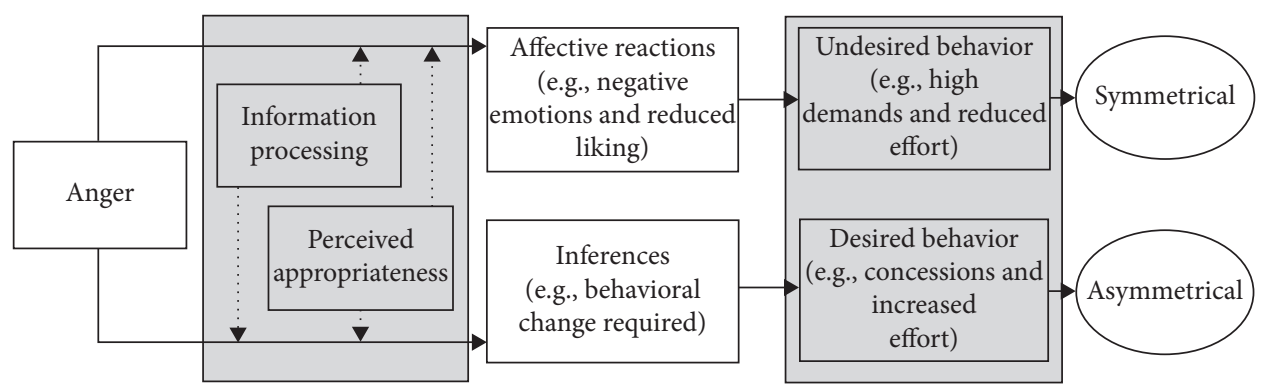

FIgURE 2: EASI model.

negative emotional state). Thus, Player 1 is the signal sender, and Player 2 is the signal receiver.

3.4. Parameter Assumptions. $W$ is the fixed salary of the miner; $A$ is the amount of fines the miner pays after a safety accident; $I$ is the safety performance salary of the miner; $S$ is the safety risk deposit paid by manager; $G$ is the fixed amount of the manager's salary; $J$ is the safety performance salary of the manager; $\Delta P$ is the change in safety performance of the emotion-driven miners after being affected by the negative emotions of the leader; $X$ is the additional damage on the emotion-driven miners' state of mind from being affected by the leader's negative emotions and, consequently, being forbidden to work downhole; $C$ is the cost of emotional supervision (such as time, money, and energy); $M$ is the cost the emotion-driven miners incur for disguising their emotional state; $M \prime$ is the cost the judgement-driven miners incur for disguising their emotional state; $\alpha$ is the probability of the emotion-driven miner causing a safety accident; $\theta$ is the safety performance transfer coefficient $(0 \leq \theta \leq 1)$; and $\varphi$ is the accident responsibility transfer coefficient $(\varphi>0)$.

3.5. Game Model. The chronological order of the game is as follows:

(1) Nature $(\mathrm{N})$ first selects the type $k \in K$ of Player 1 with type space $K=\left\{k^{E}, k^{D}\right\}$ and prior probability $P k^{D}=p$, and $P k^{E}=1-p$

(2) After observing the natural choice, Player 1 knows its type and selects one from the feasibility signal set $m \in\left\{m^{N}, m^{P}\right\}$ to send a signal

(3) After observing the signal from Player 1, Player 2 corrects the prior probability $P(k)$ using Bayes' rule to obtain the posterior probability $\widetilde{P}(k \mid m)$ of the exact type of Player 1 from the action set $a \in\left\{a^{L}, a^{N L}\right\} \quad\left(a^{L}\right.$ are the managers allowing the miners to work downhole; $a^{N L}$ are the managers not allowing the miners to work downhole)

(4) The payoffs for Player 1 and Player 2 can be expressed as $u_{1}(k, m, a)$ and $u_{2}(k, m, a)$, respectively

Based on the field investigation of a coal mine in Pingdingshan City, China, the salary standards between miners and managers were found to be quite different. Thus, the actual salary calculation method for miners and managers can be expressed as follows:

Miners' salary = basic salary + safety performance

Managers' salary = basic salary + safety performance + safety risk deposit refund

The signal game model of the miners and managers from the perspective of AET is shown in Figure 3.

\section{Game Equilibrium Analysis of the Miners and Managers from the Perspective of Emotional Events}

The perfect Bayesian equilibrium of the signal game model is a combination of a strategy combination $\left(m^{*}(k), a^{*}(k)\right)$ and posterior probability $\widetilde{P}(k \mid m)$, which needs to satisfy the following two conditions [40]:

(1) $a^{*}(m) \in u_{2}(m, a, k)$, that is, the optimal response of Player 2 to the signal sent by Player 1 in the case where the posterior probability $\widetilde{P}(k \mid m)$ is given

(2) $m^{*}(k) \in u_{1}\left(m, a^{*}(m), k\right)$, that is, the optimal strategic decision made by Player 1 in the case where the optimal response of Player 2 has been predicted

If the managers are displaying negative emotions during the prework meeting, the rational judgementdriven miners choose to display the stable emotional state, and the emotion-driven miners become infected and choose to display a negative emotional state. However, in reality, some emotion-driven miners realize that they may lose the opportunity to work downhole once their negative emotions are discovered by the managers, resulting in certain economic losses. To improve their own interests, they may disguise their real emotional state to be able to work. Suppose that, in the prework meeting, the emotion-driven miners hide their negative emotions and signal fake information with probability $\rho, 0 \leq \rho \leq 1$; that is, $P\left(m^{N} \mid k^{E}\right)=\rho, P\left(m^{P} \mid k^{D}\right)=0, P\left(m^{P} \mid k^{E}\right)=1-\rho$, and $P\left(m^{N} \mid k^{D}\right)=1$.

According to Bayes' rule, the posterior probability of Player 2 can be calculated as follows: 


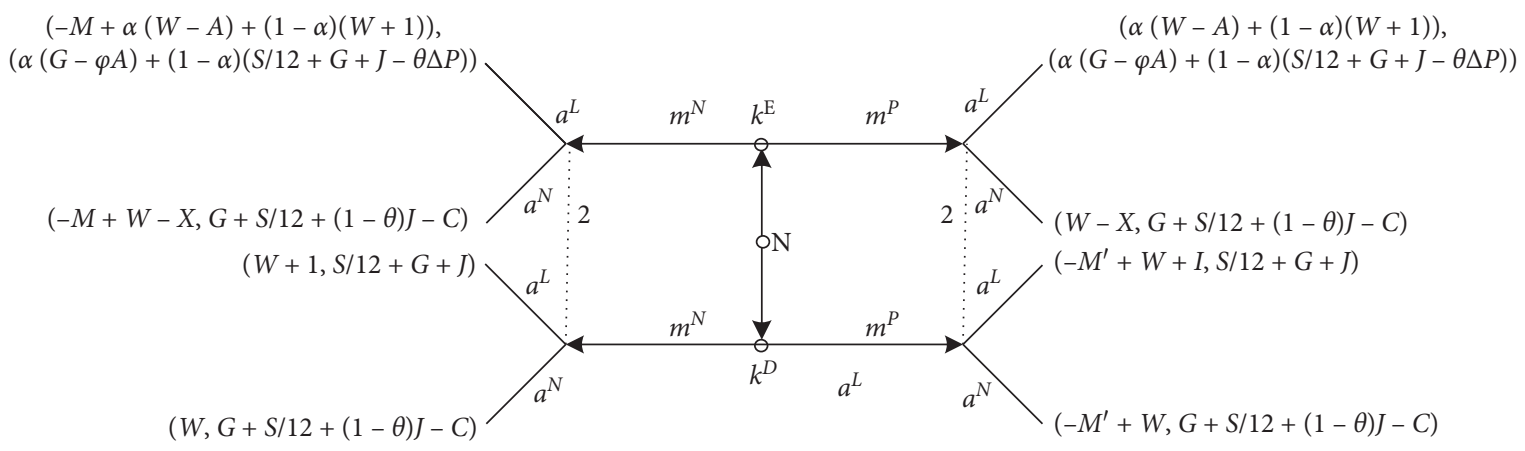

FIGURE 3: Signal game model of the miners and managers from the perspective of AET.

$$
\begin{aligned}
& \widetilde{P}\left(k^{D} \mid m^{p}\right)=\frac{P\left(k^{D}\right) \times P\left(m^{p} \mid k^{D}\right)}{P\left(m^{p}\right)}=0, \\
& \widetilde{P}\left(k^{E} \mid m^{p}\right)=\frac{P\left(k^{E}\right) \times P\left(m^{p} \mid k^{E}\right)}{P\left(m^{p}\right)}=\frac{P\left(k^{E}\right) \times P\left(m^{p} \mid k^{E}\right)}{P\left(k^{E}\right) \times P\left(m^{p} \mid k^{E}\right)+P\left(k^{D}\right) \times P\left(m^{p} \mid k^{D}\right)}=1, \\
& \widetilde{P}\left(k^{D} \mid m^{N}\right)=\frac{P\left(k^{D}\right) \times P\left(m^{N} \mid k^{D}\right)}{P\left(m^{N}\right)}=\frac{P\left(k^{D}\right) \times P\left(m^{N} \mid k^{D}\right)}{P\left(k^{E}\right) \times P\left(m^{N} \mid k^{E}\right)+P\left(k^{D}\right) \times P\left(m^{N} \mid k^{D}\right)}=\frac{P}{P+(1-P) \rho}, \\
& \widetilde{P}\left(k^{E} \mid m^{N}\right)=\frac{P\left(k^{E}\right) \times P\left(m^{N} \mid k^{E}\right)}{P\left(m^{N}\right)}=\frac{P\left(k^{E}\right) \times P\left(m^{N} \mid k^{E}\right)}{P\left(k^{E}\right) \times P\left(m^{N} \mid k^{E}\right)+P\left(k^{D}\right) \times P\left(m^{N} \mid k^{D}\right)}=\frac{(1-P) \rho}{P+(1-P) \rho} .
\end{aligned}
$$

The perfect Bayesian equilibrium of the signal game can be divided into separating equilibrium, pooling equilibrium, and quasi-separating equilibrium. According to the above, the probability $\rho$ has a significant influence on the corrected posterior probability $\widetilde{P}(k \mid m)$ of Player 2 , and the magnitude of $\rho$ has a direct significant influence on the equilibrium state of the signal game. When $\rho=0$, the signal game is in the separating equilibrium state; when $\rho=1$, the signal game is in the pooling equilibrium state; and when $0<\rho<1$, the signal game is in the quasi-separating equilibrium state. Since there are only four pure strategies in the game, the quasi-separating equilibrium can be ignored in the perfect Bayesian equilibrium; only the separating equilibrium and pooling equilibrium states will be analyzed.

\subsection{Separating Equilibrium}

Proposition 1. The signal game model has a unique separation and perfect Bayesian equilibrium. When $s<(12[(\theta-$ $\alpha) J+C-(1-\alpha) \theta \Delta P-\varphi A]) / \alpha$ is met, the emotion-driven miners choose to express negative emotions, the judgementdriven miners choose to express stable emotions, and the managers agree to let the emotion-driven miners go downhole. At this point, the regulatory system is caught in the "paradox of almost totally safe systems" [41] and is, thus, paralyzed. Proposition 1 explains the "paradox of almost totally safe systems" in the field of safety science from an economic perspective-even highly reliable organizations cannot be immune to disasters. Coal mine safety and a sound production system may become vulnerable when miners are in an unstable mood. The reason is that miners have the qualifications specified by external regulations (such as safety training) to work downhole, but their actual behaviors are largely driven by internal emotions. When the safety risk deposit paid by the manager is small enough, it cannot effectively improve the emotional supervision of the miners through economic means. The managers are likely to be either indifferent to having emotional supervision or saving on emotional supervision costs and allowing the emotion-driven miners to go downhole, the latter of which poses a safety hazard to the coal mine production. Proposition 1 also reflects that coal mining enterprises should strengthen the intangible, emotional safety management and improve the tangible, hardware conditions for safe production. Such emotional safety management methods include training programs that improve the managers' awareness and ability to provide emotional supervision and the miners' ability to recognize and handle emotions.

Proof of Proposition 1. Player 1's separating strategy includes $\left(k^{E}, k^{D}\right) \longrightarrow\left(m^{P}, m^{N}\right)$ and $\left(k^{E}, k^{D}\right) \longrightarrow\left(m^{N}, m^{P}\right)$. For the latter separating strategy $\left(k^{E}, t^{D}\right) \longrightarrow\left(m^{N}, m^{P}\right)$, there is no analytical significance in reality because emotional-driven miners may not be allowed to work downhole if they do not disguise their emotional state. At the same time, it is meaningless and counterintuitive for judgementdriven miners to disguise their stable emotional state as a negative emotional state. Therefore, this paper only discusses the first separation strategy, that is, the emotion-driven miner choosing to hide their negative emotions with 
probability $\rho=0$, leading to the separating equilibrium state in the signal game. The equilibrium strategy of the signal sender is $\left(k^{E}, k^{D}\right) \longrightarrow\left(m^{P}, m^{N}\right)$; that is, Player 1 of the $k^{E}$ type sends signal $m^{P}$, and Player 1 of the $k^{D}$ type sends signal $m^{N}$. The posterior probability of Player 2 is therefore $\widetilde{P}\left(k^{E} \mid m^{p}\right)=$ $\widetilde{P}\left(k^{D} \mid m^{N}\right)=1$, and $\widetilde{P}\left(k^{D} \mid m^{P}\right)=\widetilde{P}\left(k^{E} \mid m^{N}\right)=0$ :

(1) After Player 2 receives the signal "negative emotion" from Player 1, the expected return $E_{1}$ of selected $a^{L}$ and the expected return $E_{2}$ of selected $a^{N L}$ are, respectively,

$$
\left\{\begin{array}{l}
E_{1}=\alpha(G-\varphi A)+(1-\alpha)\left(\frac{S}{12}+G+J-\theta \Delta P\right), \\
E_{2}=G+\frac{S}{12}+(1-\theta) J-C .
\end{array}\right.
$$

According to equation (2), when $s<(12[(\theta-\alpha) J+$ $C-(1-\alpha) \theta \Delta P-\varphi A]) / \alpha, E_{1}>E_{2}$, and the expected payoffs for Player 2 allowing the miner to work downhole are higher than the payoffs for not allowing them to work downhole. Therefore, the optimal response strategy of Player 2 is allowing the miner to work downhole, $a^{*}\left(m^{p}\right)=a^{L}$. Conversely, when $s>(12[(\theta-\alpha) J+C-(1-\alpha) \theta \Delta P-\varphi A]) / \alpha$, the optimal response strategy of Player 2 is to prevent the miner from working downhole; $a^{*}\left(m^{p}\right)=a^{N L}$.

After Player 2 receives the signal "stable emotion" from Player 1 , the expected payoffs $E_{1}^{\prime}$ of selecting $a^{L}$ and expected payoffs $E_{2}^{\prime}$ of selecting $a^{N L}$ are, respectively,

$$
\left\{E_{1}^{\prime}=\frac{S}{12}+G+J, E_{2}^{\prime}=G+\frac{S}{12}+(1-\theta) J-C .\right.
$$

According to equation (3), $E_{1}^{\prime}>E_{2}^{\prime}$, which means that the expected payoffs for Player 2 allowing the miner to work downhole are higher than the payoffs for not allowing them to work downhole. The optimal strategy is thus $a^{*}\left(m^{N}\right)=a^{L}$.

(2) When the action selection for a given signal receiver is $\left(m^{P}, m^{N}\right) \longrightarrow\left(a^{L}, a^{L}\right)$, that is, $s<(12[(\theta-\alpha) J+$ $C-(1-\alpha) \theta \Delta P-\varphi A]) / \alpha$ is satisfied, the payoffs for Player 1 on the equilibrium path are

$\left\{\begin{array}{l}u_{1}\left(k^{E}, m^{P}, a^{*}\left(m^{p}\right)\right)=\alpha(W-A)+(1-\alpha)(W+I), \\ u_{1}\left(k^{D}, m^{N}, a^{*}\left(m^{N}\right)\right)=W+I .\end{array}\right.$

The payoffs for Player 1 on the nonequilibrium path are

$$
\left\{\begin{array}{l}
u_{1}\left(k^{E}, m^{N}, a^{*}\left(m^{N}\right)\right)=-M+\alpha(W-A)+(1-\alpha)(W+I), \\
u_{1}\left(k^{D}, m^{P}, a^{*}\left(m^{p}\right)\right)=-M^{\prime}+W+I
\end{array}\right.
$$

According to equations (4) and (5), $u_{1}\left(k^{D}, m^{N}\right.$, $\left.a^{*}\left(m^{N}\right)\right)>u_{1}\left(k^{D}, m^{P}, a^{*}\left(m^{P}\right)\right)$ and $u_{1}\left(k^{E}, m^{P}\right.$,
$\left.a^{*}\left(m^{p}\right)\right)>u_{1}\left(k^{E}, m^{N}, a^{*}\left(m^{N}\right)\right)$; the payoffs for Player 1 on the equilibrium path are higher than those on the nonequilibrium path. In this case, there is no incentive to deviate from equilibrium. Therefore, when the safety risk deposit paid by the manager is small enough, at $s<(12[(\theta-\alpha) J+C-(1-\alpha) \theta \Delta P-\varphi A]) / \alpha, \quad\left\{\left(m^{P}\right.\right.$, $\left.\left.m^{N}\right),\left(a^{L}, a^{L}\right),\left(\widetilde{P}\left(k^{E} \mid m^{p}\right)=1\right),\left(\widetilde{P}\left(k^{E} \mid m^{N}\right)=0\right)\right\} \quad$ is the separating perfect Bayesian equilibrium of the game.

When $s>(12[(\theta-\alpha) J+C-(1-\alpha) \theta \Delta P-\varphi A]) / \alpha$, the action selection of the given signal receiver is $\left(m^{P}, m^{N}\right) \longrightarrow\left(a^{N L}, a^{L}\right)$, and the payoffs for Player 1 on the equilibrium path are

$$
\left\{\begin{array}{l}
u_{1}\left(k^{E}, m^{P}, a^{*}\left(m^{p}\right)\right)=W-X, \\
u_{1}\left(k^{D}, m^{N}, a^{*}\left(m^{N}\right)\right)=W+I .
\end{array}\right.
$$

According to equation (6), $u_{1}\left(k^{E}, m^{P}, a^{*}\left(m^{P}\right)\right)<0$. In comparison, the expected payoffs of working downhole are positive, and, in order to maximize interests, Player 1 will disguise their emotional state and display a stable emotional state.

Proposition 1 is validated.

\subsection{Pooling Equilibrium}

Proposition 2. When the conditions $12[((C+(\theta-$ $\alpha) J-(1-\alpha) \theta \Delta P) / \alpha)-\varphi A]<s<12\{((C+J[\theta-\alpha(1-P)]) /$ $(\alpha(1-P)))+((-\alpha \varphi A+(\alpha-1) \theta \Delta P) / \alpha)\}$ and $M<\alpha(W-$ $A)+(1-\alpha)(W+I)$ are satisfied, there is only one pooling equilibrium in the signal game; that is, emotion-driven miners and judgement-driven miners show stable emotional states before work. The managers can then effectively identify the emotion-driven miners who choose the emotional contagion path and prevent them from going downhole.

The actual meaning of Proposition 2 is that when the cost incurred by the emotion-driven miners to disguise their emotional state is small enough, they choose to disguise their negative emotions in exchange for the opportunity to work downhole. In this situation, all the miners show stable emotions in the prework meeting. It is thus necessary to strengthen the managers' emotional supervision and awareness by setting a suitable safety risk deposit, thereby achieving effective supervision of the miners who choose the emotional contagion paths and, thus, have emotional safety risks.

Proof of Proposition 2. The strategies of Player 1 in the pooling equilibrium include $\left(k^{E}, k^{D}\right) \longrightarrow\left(m^{N}, m^{N}\right)$ and $\left(k^{E}, k^{D}\right) \longrightarrow\left(m^{P}, m^{P}\right)$. For the latter strategy $\left(k^{E}\right.$, $\left.k^{D}\right) \longrightarrow\left(m^{P}, m^{P}\right)$, according to the rational player hypothesis, judgement-driven miners will not disguise their stable emotional state as a negative emotional state, as it has no practical significance. Therefore, only the former kind of pooling equilibrium state is considered. When the probability that the emotion-driven miners choose to disguise their negative emotions is $1(\rho=1)$, the signal game reaches 
the pooling equilibrium state. The equilibrium strategy of the signal sender is $\left(k^{E}, k^{D}\right) \longrightarrow\left(m^{N}, m^{N}\right)$, meaning that Player 1 of the type $k^{E}$ sends signal $m^{N}$, and Player 1 of the type $k^{D}$ also sends signal $m^{N}$. The posterior probability of Player 2 is $\widetilde{P}\left(k^{D} \mid m^{N}\right)=P, \widetilde{P}\left(k^{E} \mid m^{N}\right)=1-P, \widetilde{P}\left(k^{E} \mid\right.$ $\left.m^{P}\right)=1$, and $\widetilde{P}\left(k^{D} \mid m^{P}\right)=0$ :
(1) After Player 2 receives the equilibrium signal "stable emotion" from Player 1, the expected payoffs $E_{1}^{\prime \prime}$ of the selection $a^{L}$ and the expected payoffs $E_{2}^{\prime \prime}$ of the selection $a^{N L}$ are, respectively,

$$
\left\{\begin{array}{l}
E_{1}^{\prime \prime}=\sum_{k} \widetilde{P}\left(\left(k \mid m^{N}\right) u_{2}\left(k, m^{N}, a^{L}\right)\right)=p\left(\frac{S}{12}+G+J\right)+(1-p)\left(\alpha(G-\varphi A)+(1-\alpha)\left(\frac{S}{12}+G+J-\theta \Delta P\right)\right) \\
E_{2}^{\prime \prime}=\sum_{k} \widetilde{P}\left(\left(k \mid m^{N}\right) u_{2}\left(k, m^{N}, a^{L}\right)\right)=G+\frac{S}{12}+(1-\theta) J-C
\end{array}\right.
$$

According to equation (7), if $E_{1}^{\prime \prime}>E_{2}^{\prime \prime}$, it must satisfy $s<12\left\{\frac{C+J[\theta-\alpha(1-P)]}{\alpha(1-P)}+\frac{-\alpha \varphi A+(\alpha-1) \theta \Delta P}{\alpha}\right\}$.

The expected payoffs for Player 2 choosing $a^{L}$ will be higher than the expected payoffs for choosing $a^{N L}$, so the optimal response strategy of Player 2 is $a^{*}\left(m^{N}\right)=a^{L}$. The above two conditions indicate that if the safety risk deposit is small enough when both types of miners send stable emotional signals, the managers will choose to allow both types of miners to go downhole, and the emotional supervision would have failed.

After Player 2 receives the nonequilibrium signal "negative emotion" from Player 1 , the expected payoffs $E_{1}^{\prime \prime \prime}$ of the selection $a^{L}$ and the expected payoffs $E_{2}^{\prime \prime \prime} a$ of the selection $a^{N L}$ are, respectively,

$\left\{\begin{array}{l}E_{1}^{\prime \prime \prime}=\alpha(G-\varphi A)+(1-\alpha)\left(\frac{S}{12}+G+J-\theta \Delta P\right), \\ E_{2}^{\prime \prime \prime}=G+\frac{S}{12}+(1-\theta) J-C .\end{array}\right.$

According to equation (9), if $E_{1}^{\prime \prime \prime}>E_{2}^{\prime \prime \prime}$, it is necessary to satisfy $s<12[((C+(\theta-\alpha) J-(1-\alpha) \theta \Delta P) / \alpha)-$ $\varphi A]$, and the optimal response strategy of the managers is $a^{L}$, which is $a^{*}\left(m^{P}\right)=a^{L}$; otherwise, if $E^{\prime \prime \prime}{ }_{1}<E^{\prime \prime \prime}{ }_{2}, \quad s>12[((C+(\theta-\alpha) J-(1-\alpha) \theta \Delta P) / \alpha)-$ $\varphi A]$, and the optimal response strategy for the managers is $a^{N L}$, which is $a^{*}\left(m^{P}\right)=a^{N L}$.

(2) When the optimal selection strategy for a given Player 2 is $\left(m^{N}, m^{P}\right) \longrightarrow\left(a^{L}, a^{L}\right)$, it is necessary to satisfy the conditions $s<\min (12\{((C+J[\theta-\alpha(1-$ $P)]) /(\alpha(1-P)))+\quad((-\alpha \varphi A+(\alpha-1) \theta \Delta P) / \alpha)\}$, and $s<12[((C+(\theta-\alpha) J-(1-\alpha) \theta \Delta P) / \alpha)-\varphi A])$, and the payoffs for Player 1 on the equilibrium path are as follows:

$\left\{\begin{array}{l}u_{1}\left(k^{E}, m^{N}, a^{*}\left(m^{N}\right)\right)=-M+\alpha(W-A)+(1-\alpha)(W+I), \\ u_{1}\left(k^{D}, m^{N}, a^{*}\left(m^{N}\right)\right)=W+I .\end{array}\right.$
The payoffs for Player 1 on the nonequilibrium path are as follows:

$$
\left\{\begin{array}{l}
u_{1}\left(k^{E}, m^{P}, a^{*}\left(m^{P}\right)\right)=W-X, \\
u_{1}\left(k^{D}, m^{P}, a^{*}\left(m^{P}\right)\right)=-M^{\prime}+W .
\end{array}\right.
$$

It is assumed in the model hypothesis that $W-X<0$, and the expected payoffs for the miners working downhole are positive; thus, it is only necessary to ensure that $u_{1}\left(k^{E}, m^{N}, a^{*}\left(m^{N}\right)\right)>0$, and the payoffs for Player 1 on the equilibrium path are higher than those on the nonequilibrium path.

Therefore, when the condition satisfies $12[((C+)(\theta-$ $\alpha) J-(1-\alpha) \theta \Delta P) / \alpha)-\varphi A]<s<12\{((C+J[\theta-$ $\alpha(1-P)]) /(\alpha(1-P)))+((-\alpha \varphi A+(\alpha-1) \theta \Delta P) / \alpha)\}$ and $\quad M<\alpha(W-A)+(1-\alpha)(W+I)$, then $\left\{\left(k^{E}, k^{D}\right),\left(m^{N}, m^{N}\right),\left(a^{N L}, a^{L}\right)\right\}$ is the pooling perfect Bayesian equilibrium of the signal game.

Proposition 2 is validated.

\section{Real Case Demonstration}

The selected case study belongs to the China Pingmei Shenma Group. The coal mine was officially opened in February 1964 and generated an annual output of 2.9 million tons of coal, bringing more than 20 million yuan in annual tax revenue to the local government. The geological conditions of the coal mine are complex; a typical underground coal mine requires the coal seam to be buried relatively deep.

The values of the parameters are mainly obtained from the official announcements of the coal mining enterprise and further investigations. Due to the fluctuation of some values within a certain range, the mean values are adopted for simplicity. Table 1 shows the value assignment of the parameters.

5.1. Separating Equilibrium. Once the values of the above parameters are substituted into equations (2)-(6), it can be concluded that when the safety risk deposit paid by the managers is $S<2,400$, the separation equilibrium occurs. In this situation, the managers fail to realize effective emotional supervision, resulting in the failure of the safety risk deposit system. 
TABLE 1: Parameter settings for the case analysis.

\begin{tabular}{lc}
\hline Parameter & Value \\
\hline$W$ & 4,000 \\
$A$ & 800 \\
$I$ & 800 \\
$G$ & 6,000 \\
$J$ & 1,200 \\
$\Delta P$ & 400 \\
$X$ & 80 \\
$C$ & 600 \\
$\alpha$ & 0.5 \\
$\theta$ & 0.5 \\
$\varphi$ & 0.5 \\
\hline
\end{tabular}

5.2. Pooling Equilibrium. Assuming $1-p=0.5$, which means the probability of the emotion-driven miners exhibiting a stable emotional state is 0.5 , and substituting the above parameter values into equations (7)-(11), it can be concluded that when $7,200<S<36,000$ and $M<4,000$, the system reaches a pooling equilibrium; the safety risk deposit system works, and the managers are motivated to perform effective emotional supervision of miners' emotional states.

However, when $2,400<S<7,200$ or $S>36,000$, the separating equilibrium and pooling equilibrium coexist, and the system falls into chaos. According to the investigated data, the average safety risk deposit of this coal mine is 12,000 , which is within a rational range.

\section{Conclusions and Implications}

A signal game model on emotional supervision is constructed from the AET perspective, the separating equilibrium and pooling equilibrium states are analyzed, and the perfect Bayesian equilibrium paths of the two equilibrium states are obtained. The research not only proves that the repeated emergence of coal mine safety accidents is the consequence of "the paradox of almost totally safe systems" but also explains the reason for this paradox from an economic perspective. When the amount of safety risk deposit paid by manager is small enough, the economic constraint may not motivate the managers to improve their awareness and ability to provide effective emotional supervision. In this situation, the managers only consider the miners' external, formalistic safety conditions (such as qualifications and health condition), which are easy to obtain, and ignore their internal, emotional safety states. Thus, the miners with hidden emotional safety risks may pose a risk to the mining enterprise's operations. Consequently, when the cost of disguising their emotional state is small enough, the emotion-driven miners may choose to disguise negative emotions in order to be able to work downhole. Therefore, effective economic constraints should be formed by setting a suitable safety risk deposit to strengthen emotional supervision and creating punitive measures to prevent miners from disguising their emotions.

\section{Data Availability}

No data were used to support this study.

\section{Conflicts of Interest}

The authors declare that there are no conflicts of interest regarding the publication of this paper.

\section{Authors' Contributions}

All authors contributed equally to this work.

\section{Acknowledgments}

This work was supported by the National Natural Science Foundation of China (Grant no. 71573086), the Henan Province Water Resources Security and Clean Energy Collaborative Management Innovative Science and Technology Team Project, and the Doctoral Innovation Fund of North China University of Water Resources and Electric Power.

\section{References}

[1] P. K. Piff and J. P. Moskowitz, "Wealth, poverty, and happiness: social class is differentially associated with positive emotions," Emotion, vol. 18, no. 6, pp. 902-905, 2017.

[2] M. J. Sanchez-Ruiz, C. El-Jor, J. Abi Kharma, M. Bassil, and N. Zeeni, "Personality, emotion-related variables, and media pressure predict eating disorders via disordered eating in Lebanese university students," Eating and Weight DisordersStudies on Anorexia, Bulimia and Obesity, vol. 24, no. 2, pp. 313-322, 2017.

[3] J. Tang, "Emotion and security research," Electric Safety Technology, vol. 15, no. 7, pp. 8-10, 2003.

[4] J. M. Roche and H. S. Arnold, "The effects of emotion suppression during language planning and production," Journal of Speech Language and Hearing Research, vol. 61, no. 8, pp. 1-8, 2018.

[5] A. D. Gershoff and J. J. Koehler, "Safety first? the role of emotion in safety product betrayal aversion," Journal of Consumer Research, vol. 38, no. 1, pp. 140-150, 2011.

[6] M. Aranguren, "Emotional mechanisms of social (re)production," Social Science Information, vol. 54, no. 4, pp. 543563, 2015.

[7] Z. Ruida, X. Zhenhua, T. Honghong et al., "The effect of shame on anger at others: awareness of the emotion-causing events matters," Cognition and Emotion, vol. 33, no. 4, pp. 1-13, 2019.

[8] R. Cropanzano and M. T. Dasborough, "Dynamic models of well-being: implications of affective events theory for expanding current views on personality and climate," European Journal of Work and Organizational Psychology, vol. 24, no. 6, pp. 844-847, 2015.

[9] M. M. Luo and S. Chea, "Cognitive appraisal of incident handling, affects, and post-adoption behaviors: a test of affective events theory," International Journal of Information Management, vol. 40, pp. 120-131, 2018.

[10] H. M. Weiss and R. Cropanzano, "Affective events theory: a theoretical discussion of the structure, causes and consequences of affective experiences at work," Research in Organizational Behavior, vol. 18, no. 3, pp. 1-74, 1996.

[11] C. R. Beasley and L. A. Jason, "Engagement and disengagement in mutual-help addiction recovery housing: a test of affective events theory," American Journal of Community Psychology, vol. 55, no. 3-4, pp. 347-358, 2015. 
[12] W. Lam and Z. Chen, "When I put on my service mask: determinants and outcomes of emotional labor among hotel service providers according to affective event theory," International Journal of Hospitality Management, vol. 31, no. 1, pp. 3-11, 2012.

[13] D. A. Drury, S. A. Ferguson, and M. J. W. Thomas, "Restricted sleep and negative affective states in commercial pilots during short haul operations," Accident Analysis \& Prevention, vol. 45 , no. 1 , pp. 80-84, 2012.

[14] K. M. Kowalski-Trakofler and E. A. Barrett, "The concept of degraded images applied to hazard recognition training in mining for reduction of lost-time injuries," Journal of Safety Research, vol. 34, no. 5, pp. 515-525, 2003.

[15] J. A. Russell, "Is there universal recognition of emotion from facial expression? a review of the cross-cultural studies," Psychological Bulletin, vol. 115, no. 1, pp. 102-141, 1994.

[16] Y. Sui, R. Ding, and H. Wang, "An integrated management system for occupational health and safety and environment in an operating nuclear power plant in East China and its management information system," Journal of Cleaner Production, vol. 183, pp. 261-271, 2018.

[17] J. Qijun and P. J. Batt, "Barriers and benefits to the adoption of a third party certified food safety management system in the food processing sector in Shanghai, China," Food Control, vol. 62, pp. 89-96, 2016.

[18] Z. Y. Li, "Strive to improve the safety production level of coal mines (EB/OL)," National Coal Mine Safety Supervision Administration, China, 2019, http://www.chinacoal-safety. gov.cn/xw/mkaqjexw/201901/t20190125_224053.shtml.

[19] J. Chen, K. Milne, J. Dayman, and E. Kemps, "Interpretation bias and social anxiety: does interpretation bias mediate the relationship between trait social anxiety and state anxiety responses?" Cognition and Emotion, vol. 33, no. 4, pp. 630$645,2019$.

[20] B. Mareike and S. Georges, "'Effects of habitual anger on employees' behavior during organizational change," International Journal of Environmental Research and Public Health, vol. 10, no. 12, pp. 6215-6234, 2013.

[21] E. Lahav, T. Shavit, and U. Benzion, "Can't wait to celebrate: holiday euphoria, impulsive behavior and time preference," Journal of Behavioral and Experimental Economics, vol. 65, pp. 128-134, 2016.

[22] S.-D. Martin, "Fear and anxiety while driving: differential impact of task demands, speed and motivation," Transportation Research Part F: Traffic Psychology and Behaviour, vol. 16, pp. 14-28, 2013

[23] D. Shinar, "Aggressive driving: the contribution of the drivers and the situation," Transportation Research Part F: Traffic Psychology and Behaviour, vol. 1, no. 2, pp. 137-160, 1998.

[24] L. L. Di Stasi, D. Contreras, J. J. Cañas, A. Cándido, A. Maldonado, and A. Catena, "The consequences of unexpected emotional sounds on driving behaviour in risky situations," Safety Science, vol. 48, no. 10, pp. 1463-1468, 2010.

[25] L. O. Lundqvist and U. Dimberg, "Facial expressions are contagious," Journal of Psychophysiology, vol. 9, no. 3, pp. 203-211, 1995.

[26] M. L. Hoffman, "How automatic and representational is empathy, and why," Behavioral \& Brain Sciences, vol. 25, no. 1, pp. 38-39, 2001.

[27] E. Hatfreld, J. T. Cacioppo, and R. L. Rapson, "Emotional contagion," Current Directions in Psychological Science, vol. 2, no. 3, pp. 96-99, 1993.

[28] J. N. Choi, S. Y. Sung, K. Lee, and D.-S. Cho, "Balancing cognition and emotion: innovation implementation as a function of cognitive appraisal and emotional reactions toward innovation," Journal of Organizational Behavior, vol. 32, no. 1, pp. 107-124, 2011.

[29] J. A. Bunk and V. J. Magley, "The role of appraisals and emotions in understanding experiences of workplace incivility," Journal of Occupational Health Psychology, vol. 18, no. 1, pp. 87-105, 2013.

[30] S. Lim and A. Lee, "Work and nonwork outcomes of workplace incivility: does family support help?" Journal of Occupational Health Psychology, vol. 16, no. 1, pp. 95-111, 2011.

[31] R. S. Lazarus, "Progress on a cognitive-motivational-relational theory of emotion," American Psychologist, vol. 46, no. 8, pp. 819-834, 1991.

[32] A. M. Game, "Workplace boredom coping: health, safety, and HR implications," Personnel Review, vol. 36, no. 5, pp. 701-721, 2007.

[33] J. B. Rodell and T. A. Judge, "Can "good" stressors spark "bad" behaviors? The mediating role of emotions in links of challenge and hindrance stressors with citizenship and counterproductive behaviors," Journal of Applied Psychology, vol. 94, no. 6, pp. 1438-1451, 2009.

[34] J. A. Hansen and S. Pihl-Thingvad, "Managing employee innovative behaviour through transformational and transactional leadership styles," Public Management Review, vol. 21, no. 6, pp. 918-944, 2019.

[35] J. Wegge, R. V. Dick, G. K. Fisher, M. A. West, and J. F. Dawson, "A test of basic assumptions of affective events theory (AET) in call centre Work 1," British Journal of Management, vol. 17, no. 3, pp. 237-254, 2006.

[36] D. Lindebaum, P. J. Jordan, and M. T. Dasborough, “"When it can be good to feel bad, and bad to feel good-exploring asymmetries in workplace emotional outcomes," Human Relations, vol. 66, no. 1, pp. 154-156, 2013.

[37] V. A. Visser, D. Van Knippenberg, G. A. Van Kleef, and B. Wisse, "How leader displays of happiness and sadness influence follower performance: emotional contagion and creative versus analytical performance," The Leadership Quarterly, vol. 24, no. 1, pp. 172-188, 2013.

[38] G. A. Kleef, "Understanding the positive and negative effects of emotional expressions in organizations: EASI does it," Human Relations, vol. 67, no. 9, pp. 1145-1164, 2014.

[39] Y.-H. Hsieh and S.-Y. Yeh, "Modeling dynamic service recovery strategies: a signaling game approach," Kybernetes, vol. 47, no. 5, pp. 888-919, 2018.

[40] L. Zhao and D. Li, "Analysis and control of the complex dynamics of a multimarket cournot investment game with bounded rationality," Discrete Dynamics in Nature and Society, vol. 2016, no. 2, 10 pages, 2016.

[41] N. Oliver, T. Calvard, and K. Potočnik, "Cognition, technology, and organizational limits: lessons from the Air France 447 disaster," Organization Science, vol. 28, no. 4, pp. 729-743, 2017. 